Polymer Journal, Vol. 3, No. 3, pp 370-377 (1972)

\title{
Kinetic Aspects of the Alkaline Hydrolysis of Poly(acrylamide)
}

\author{
Mitsuo Higuchi and Ryoichi Senju \\ Department of Forest Products, Faculty of Agriculture, \\ Kyushu University, Fukuoka, Japan.
}

(Received September 25, 1971)

\begin{abstract}
The alkaline hydrolysis of poly(acrylamide) was followed by determining the residual amount of unreacted amide group.

Contrary to the report of Moens and Smets, the plots of the reaction based on the simple second-order rate equation form smooth curves showing no distinction for steps involved, and a kind of conversion limit exists when hydrolysis is about $60 \%$ complete.

Theoretical calculations based on the assumption that the reactivity of each amide group depends upon whether zero, one, or two of its nearest neighbors have reacted were compared with the experimental data. Under the ionic strength of about 0.5 , the actual progress of the reaction is in satisfactory accord with the theoretical figure.

KEY WORDS Poly(acrylamide) / Alkaline Hydrolysis / Electrostatic

Repulsion Effect / Conversion Limit / Statistical Calculation /
\end{abstract}

Alkaline hydrolysis of poly(vinylamides) ${ }^{1-3}$ is one of the ideal subjects of study on the effects of neighboring groups in the reaction of chain molecules, as it can be followed over its entire course in a single solvent medium (aqueous medium). On this subject, however, no satisfactorily quantitative investigation has been reported. This may be attributed to the inaccuracy of experimental data. For instance, it has been reported by Moens and Smets $^{3}$ that the firstorder plot of the hydrolysis of poly(acrylamide) in the presence of a large excess of sodium hydroxide $(0.5 \mathrm{~N})$ is divided into two distinct lines, i.e., the reaction involves two different steps. On the other hand, in the case of poly(methacrylamide) the rate constant decreases progressively as the conversion increases. On the basis of these observations, Smets emphasized the role of polymer structure in the reaction of chain molecules. However, their results, especially on poly(acrylamide), are problematical. Moens and Smets adopted conductometric titration for determination of the degree of hydrolysis. Conductometric titration is, however, not suitable for determination of polymer electrolytes because of the obscurity of the critical points in the titration curve; and, particularly in the case where a large excess of alkali is present in the reaction mixture, the obtained value for carboxyl content involves large error.

By the way, Keller ${ }^{4}$ and others ${ }^{4}$ have investigated the kinetic problem of neighboring group effects for long-chain molecules. They derived general kinetic expressions for the entire course of the process assuming that the reactivity of each group depends upon whether zero, one, or two of its nearest neighbors have reacted. However, no fruitful comparison of theoretical calculations with experimental data has yet been achieved.

We consider that the alkaline hydrolysis of poly(vinylamide)s will conform to the theoretical figure provided that the reaction condition is suitable. For evidence, some new precise experimental data on poly(acrylamide) are presented in this paper, and they are compared with theoretical calculations.

The equations derived by Keller or others are, however, not always convenient for analysis of experimental data; in those equations the average fractions of the reacted group and unreacted groups of the three species are expressed as functions of time. On that account, first we consider the problem from a different angle to facilitate the evaluation of conversion limit and rate constants for three-classified amide groups. 
Kinetic Aspects of the Alkaline Hydrolysis of Poly(acrylamide)

\section{THEORETICAL}

\section{Statistical Calculation of the Conversion Limit}

It may be assumed that the electroestatic repulsion between a carboxyl group and catalyzing hydroxyl ions is effective only in the immediate vicinity of the ionized carboxyl. For the reactions under those conditions we can divide the amide groups in a molecule into the following three classes.

I: amide groups whose nearest neighbors have not reacted.

II: amide groups which have one neighbor entering into reaction.

III: amide groups which have two neighbors entering into reaction.

Let $k_{\mathrm{I}}, k_{\mathrm{II}}$, and $k_{\mathrm{III}}$ denote the rate constants for the groups I, II, and III, respectively. Since the order of the rate constants is considered to be $k_{\mathrm{I}}>k_{\mathrm{II}}>k_{\mathrm{III}}$, the progress of the reaction may be shown schematicaly as Figure 1. Figure 1a corresponds to the early stage of the reaction, where the greater part of the reaction is carried out by the group I. Figure $1 \mathrm{~b}$ shows the situation at the point where the group I has disappeared, and from this state almost all of the reaction is attributed to the group II. Figure 1c shows the situation at the point where the group II has disappeared. After reaching the state (c), if $k_{\mathrm{III}}$ be sufficiently smaller than $k_{\mathrm{II}}$, the velocity of the reaction will diminish conspicuously to show a kind of conversion limit.
If it be assumed that $k_{\text {III }}$ is equal to zero, values for the conversion limit can be calculated in the following manner. Let us denote by $S_{n}$ the number of the amide groups which will be left unreacted in a polymer chain of polymerization degree $n$ at the termination of the reaction in the case where the first reaction occurs at one of the end groups, and denote by $Z_{n}$ that in the case where the reaction starts from the state where both of the end groups have already reacted. It is convenient to assign a number to each group, $1,2,3, \ldots n$, from one end of a chain to the other.

In the case where No. 1 reacted first

$$
12345 \cdots n
$$

number of the remainder at the termination of the reaction is $S_{n}$ according to the definition.

In the case where No. 2 reacted first

$$
\underbrace{12}_{2} \underbrace{2300 \cdots \cdots}_{n-1}
$$

the chain can be divided into two parts, both have one reacted end group at the start of the reaction. Hence, the number of the remainder in this case is equal to the sum of $S_{2}$ and $S_{n-1}$. In the general case where No. $i$ reacted first

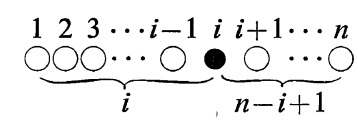

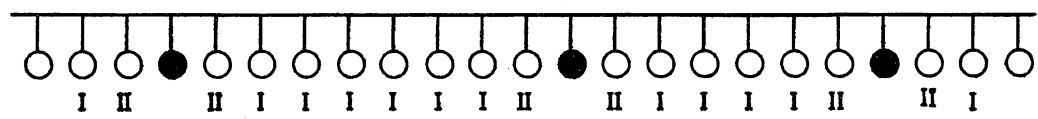

(b)
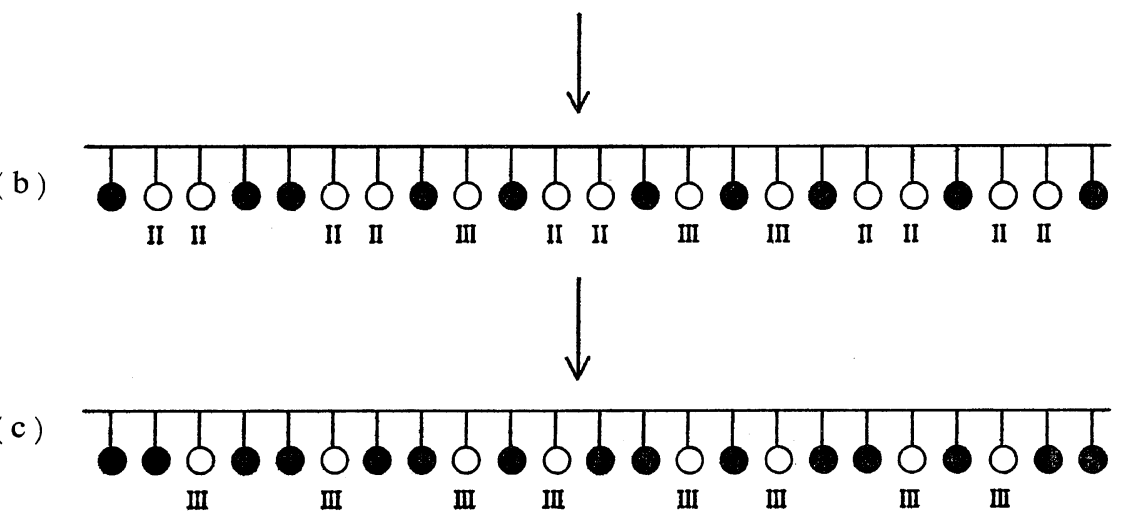

Figure 1. Schematic aspect of the progess of the reaction. 


\section{Higuchi and R. Senju}

the number of the remainder is equal to the sum of $S_{i}$ and $S_{n-i+1}$ according to the reason described above.

The probability of an amide group reacting first in a chain is equal to $1 / n$, because the location of the first reaction of amide groups in a chain is considered to be a matter of chance. Hence, for a group of polymer molecules, each containing $n$ structural units, the average number of the remainder per molecule, $\bar{S}_{n}$, is given by

$$
\bar{S}_{n}=\frac{2}{n}\left(S_{2}+S_{3}+S_{4}+\ldots+S_{n}\right)
$$

Our next step is to consider $S_{n}$. Consider a chain containing $n$ structural units, No. 1 of which has already reacted.

In the case where the second reaction occured at No. 2

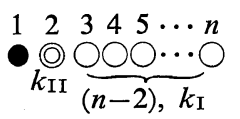

the number of the remainder in this case is equal to $S_{n-1}$, and the probability of No. 2 reacting next to No. 1 is considered to be equal to $k_{\mathrm{II}} /\left[(n-2) k_{\mathrm{I}}+k_{\mathrm{II}}\right]$.

In the case where the second reaction occured at No. 3

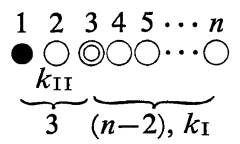

we can divide the chain into two parts. One has two reacted end groups, and another has one reacted end group at the start of the reaction. Hence, the number of the remainder in this case is equal to the sum of $Z_{3}$ and $S_{n-2}$. And the probability of No. 3 reacting next to No. 1 is equal to $k_{\mathrm{I}} /\left[(n-2) k_{\mathrm{I}}+k_{\mathrm{II}}\right]$.

Continuing in this manner, we obtain an expression for $S_{n}$.

$$
\begin{aligned}
S_{n}= & \frac{k_{\mathrm{II}}}{(n-2) k_{\mathrm{I}}+k_{\mathrm{II}}} S_{n-1} \\
& +\frac{k_{\mathrm{I}}}{(n-2) k_{\mathrm{I}}+k_{\mathrm{II}}}\left(\sum_{r=2}^{n-2} S_{r}+\sum_{r=3}^{n} Z_{r}\right)
\end{aligned}
$$

In the same manner we obtain the equation for $Z_{n}$.

$$
\begin{aligned}
Z_{n}= & \frac{2 k_{\mathrm{II}}}{(n-4) k_{\mathrm{I}}+2 k_{\mathrm{II}}} Z_{n-1} \\
& +\frac{2 k_{\mathrm{I}}}{(n-4) k_{\mathrm{I}}+2 k_{\mathrm{II}}} \sum_{r=3}^{n-2} Z_{r}
\end{aligned}
$$

Now we can calculate any optional $\bar{S}_{n}$ by the use of eq 1,2, and 3. The terms at the start of the calculation are: $Z_{3}=1, S_{2}=0$ (when $k_{\mathrm{II}}$ is not equal to zero) or $S_{2}=1$ (when $k_{\mathrm{II}}$ is equal to zero).

This type of series, $\bar{S}_{2}, \bar{S}_{3}, \bar{S}_{4}, \ldots \bar{S}_{i}$, becomes, fortunately, an arithmetical progression beyond fifteen or twenty terms, and can be expressed by

$$
\bar{S}_{n}=S_{i}+\delta(n-i)
$$

and one can readily obtain the value for $\delta$ by calculating only twenty terms or thereabouts in the progression.

The conversion limit, $R$, is given by

$$
R=1-\bar{S}_{n} / n
$$

Hence, for a high-molecular-weight polymer where $n$ is sufficiently large

$$
R \cong 1-\delta
$$

Values for $R$ thus obtained for various combinations of $k_{\mathrm{I}}$ and $k_{\mathrm{II}}$ are listed in Table I.

Table I. Calculated values for conversion limit

\begin{tabular}{cccc}
\hline$k_{\mathrm{II}} / k_{\mathrm{I}}$ & \multicolumn{1}{c}{$R$} & $k_{\mathrm{II}} / k_{\mathrm{I}}$ & \multicolumn{1}{c}{$R$} \\
\hline $1 / 1$ & 0.6667 & $1 / 5$ & 0.6001 \\
$1 / 2$ & 0.6321 & $1 / 10$ & 0.5856 \\
$1 / 3$ & 0.6160 & $1 / 20$ & 0.5771 \\
$1 / 4$ & 0.6065 & $1 / 100$ & 0.5697 \\
\hline
\end{tabular}

Relations between Reaction Velocity and Degree of Conversion

In this most simplified case where only the nearest neighboring amide groups are affected by carboxyl groups, and, therefore, the amide groups are divided into three classes, I, II, and III, the reaction rate is given by

$$
\begin{aligned}
\frac{\mathrm{d} x}{\mathrm{~d} t} & =\frac{-\mathrm{d}\left[-\mathrm{CONH}_{2}\right]}{\mathrm{d} t}=\frac{\mathrm{d}\left[-\mathrm{COO}^{-}\right]}{\mathrm{d} t} \\
& =\left(k_{\mathrm{I}}[\mathrm{I}]+k_{\mathrm{II}}[\mathrm{II}]+k_{\mathrm{III}}[\mathrm{III}]\right)\left[\mathrm{OH}^{-}\right]
\end{aligned}
$$

If it be assumed that $k_{\text {III }}$ is equal to zero, the average fractions of I, II, and III, as functions of the degree of conversion are obtainable, as visualized in Figure 2. Curves in Figure 2 
Kinetic Aspects of the Alkaline Hydrolysis of Poly(acrylamide)

are obtained in the following manner. First, consider the case where the group II does not react until the group I disappears and the group III is not reactable, i.e., $k_{\mathrm{I}} \gg k_{\mathrm{II}} \gg k_{\mathrm{III}}=0$. The value for the degree of conversion, 0.432 , at which the group I disappears, is obtained by the calculation of the conversion limit $(R) ; k_{\mathrm{II}}=0$ being put into eq 2 and 3 . The value for the degree of conversion, 0.568 , at which the group II disappears, is obtained as a limiting value, as $k_{\mathrm{II}} / k_{\mathrm{I}}$ approaches zero, for $R$. Obviously, $[\mathrm{I}]+[\mathrm{II}]+[\mathrm{III}]+\left[-\mathrm{COO}^{-}\right]=$const, and in the early stage of the reaction $-\mathrm{d}[\mathrm{I}] / \mathrm{d} x=3$, and $\mathrm{d}[\mathrm{II}] / \mathrm{d} x=2$, where $x$ is the concentration of the reacted group. In the range of the degree of conversion $0.432 \sim 0.568,-\mathrm{d}[\mathrm{II}] / \mathrm{d} x=2$, and d[III]/ $\mathrm{d} x=1$. [III] in the early stage of the reaction is obtained by calculation of probabilities. According to these relationships, the solid curves in Figure 2 are drawn. The broken curves and dotted ones correspond to the cases where $k_{\mathrm{II}} / k_{\mathrm{I}}$ is equal to $1 / 5$ and $1 / 3$, respectively, and these are obtained by numerical calculations.

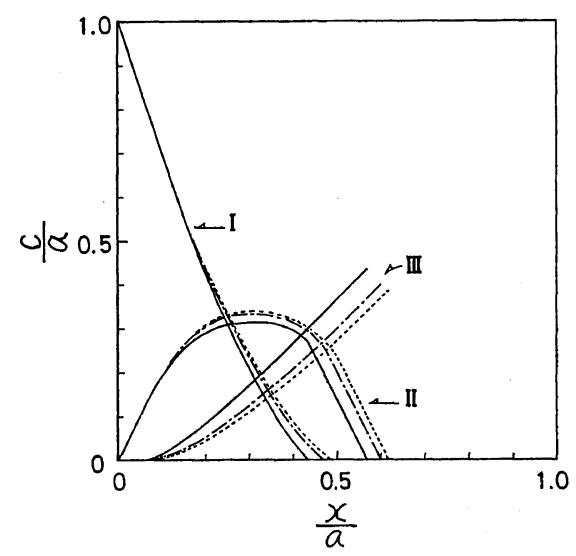

Figure 2. Relations between conversion degree and average fractions of I, II, and III: - $k_{\mathrm{I}} \gg k_{\mathrm{II}}$ $\gg k_{\mathrm{III}}=0 ;--\longrightarrow, k_{\mathrm{I}}: k_{\mathrm{II}}: k_{\mathrm{III}}=1: 1 / 5: 0 ;-\cdots$, $k_{\mathrm{I}}: k_{\mathrm{II}}: k_{\mathrm{III}}=1: 1 / 3: 0$.

We see that concentrations of I and II in the early stage of the reaction can be approximately expressed by

$$
\begin{aligned}
{[\mathrm{I}] } & =a-3 x \\
{[\mathrm{II}] } & =2 x
\end{aligned}
$$

where $a$ is the initial concentration of amide group. And, in the range, from $R_{1}$, where the group I disappears, to $R_{2}$, where the group II disappears, it is shown by

$$
[\mathrm{II}]=2\left(a R_{2}-x\right)
$$

Hence, we obtain a expression for the rate of the reaction in the early stage as

$$
\frac{\mathrm{d} x}{\mathrm{~d} t}=k_{\mathrm{I}}\left[a-\left(3-\frac{2 k_{\mathrm{II}}}{k_{\mathrm{I}}}\right) x\right]\left[\mathrm{OH}^{-}\right]
$$

and in the range, $R_{1} \sim R_{2}$, as

$$
\frac{\mathrm{d} x}{\mathrm{~d} t}=2 k_{\mathrm{II}}\left(a R_{2}-x\right)\left[\mathrm{OH}^{-}\right]
$$

\section{EXPERIMENTAL WORK}

\section{Material}

Polymerization of acrylamide was carried out in $15-\%$ aqueous solution with the use of potassium persulfate-isopropanol redox initiator under $\mathrm{N}_{2}$ atmosphere, at $60^{\circ} \mathrm{C}$, for $60 \mathrm{~min}$. Nitrogen content of the polymer was $19.56 \%$. Carboxyl content was $1.3 \times 10^{-4} \mathrm{~mol}$ per $1 \mathrm{~g}$ of polymer; the ratio $-\mathrm{COOH} /-\mathrm{CONH}_{2}$ is below 0.001 . The intrinsic viscosity, $[\eta]$, of the polymer in water was $2.3 \mathrm{dl} / \mathrm{g}$ at $30^{\circ} \mathrm{C}$.

\section{Hydrolysis Rate Measurement}

The hydrolysis reactions at low temperatures $\left(30^{\circ} \sim 50^{\circ} \mathrm{C}\right)$ were carried out in a $500-\mathrm{m} l$ threenecked flask equipped with a mercury sealed stirrer, and immersed in a constant temperature bath $\left( \pm 0.01^{\circ} \mathrm{C}\right)$. A $10-\mathrm{m} l$ portion of the reaction mixture was pipetted out and analyzed for a residual amount of amide groups at moderate intervals. In the cases of the reactions at high temperatures $\left(60 \sim 100^{\circ} \mathrm{C}\right)$, sodium hydroxide solution was mixed with the polymer solution at $0^{\circ} \mathrm{C}$, and was transferred into $15-\mathrm{m} l$ pyrex ampoules. Then the ampoules were immersed in a constant-temperature bath.

The degree of conversion was determined by precipitating the whole polymer from the pipetted reaction mixture and analyzing the polymer for nitrogen content by Kjeldahl method. Since the accuracy of the analysis by this procedure was dependent upon complete precipitation of the polymer and complete removal of ammonia from the precipitated matter, various precipitants for partially hydrolyzed poly(acrylamide) and operat- 


\section{Higuchi and R. Senju}

ing conditions were exaimed. Best results were obtained by the following procedure.

A $10-\mathrm{m} l$ portion of the reaction mixture was pipetted out and transferred into a $200-\mathrm{m} l$ beaker containing $50 \mathrm{~m} l$ of dioxane, $40 \mathrm{~m} l$ of water, and $1 \mathrm{ml}$ of acetic acid. The mixture solution was agitated by a magnetic stirrer, and $35 \mathrm{ml}$ of dioxane was added to precipiate the polymer. Agitation was continued until the supernatant fluid became completely transparent. Then the liquid phase was removed, and the precipitate was dissolved with $10 \mathrm{ml}$ of $1-\%$ aqueous sodium carbonate solution and reprecipitated by the addition of $100 \mathrm{ml}$ of $70-\%$ dioxane. After completion of the precipitation the supernatant fluid was removed, and the polymer precipitate was dissolved with a small amount of $1-\%$ aqueous sodium carbonate solution and transferred into a Kjeldahl flask by repeated washing with small amounts of water. Then the polymer was analyzed by Kjeldahl method.

\section{RESULTS AND DISCUSSION}

Results are listed in Tables II $\sim$ IV.

\section{Simple Pseudo-First-Order Plots}

Contrary to the report of Moens and Smets, the plots of the authors' data form smooth curves, as can be seen in Figure 3, showing no distinction for the steps involved.

The phenomenon that the plot based on a simple second-order rate equation is divided into

Table II. Hydrolysis of poly(acrylamide) ${ }^{a}$

\begin{tabular}{|c|c|c|c|c|c|}
\hline \multicolumn{2}{|c|}{$30^{\circ} \mathrm{C}$} & \multicolumn{2}{|c|}{$40^{\circ} \mathrm{C}$} & \multicolumn{2}{|c|}{$50^{\circ} \mathrm{C}$} \\
\hline$t, \min$ & $x / a$ & $t, \min$ & $x / a$ & $t, \min$ & $x / a$ \\
\hline 15 & 0.0229 & 5 & 0.0172 & 5 & 0.0331 \\
\hline 30 & 0.0426 & 10 & 0.0336 & 10 & 0.0675 \\
\hline 45 & 0.0621 & 15 & 0.0491 & 20 & 0.1238 \\
\hline 60 & 0.0787 & 20 & 0.0631 & 30 & 0.1660 \\
\hline 90 & 0.1095 & 30 & 0.0905 & 45 & 0.2145 \\
\hline 120 & 0.1379 & 45 & 0.1249 & 60 & 0.2547 \\
\hline 170 & 0.1765 & 65 & 0.1626 & 80 & 0.2928 \\
\hline 240 & 0.2191 & 90 & 0.2023 & 110 & 0.3350 \\
\hline 330 & 0.2617 & 120 & 0.2383 & 150 & 0.3739 \\
\hline 450 & 0.3014 & 160 & 0.2752 & 200 & 0.4079 \\
\hline 600 & 0.3355 & 210 & 0.3108 & 260 & 0.4352 \\
\hline 780 & 0.3671 & 270 & 0.3423 & 330 & 0.4567 \\
\hline 990 & 0.3935 & 340 & 0.3702 & 410 & 0.4749 \\
\hline 1230 & 0.4178 & 420 & 0.3948 & & \\
\hline 1500 & 0.4381 & 510 & 0.4169 & & \\
\hline
\end{tabular}

a $\mathrm{NaOH}, 0.25 \mathrm{~mol} / l$; amide, $0.025 \mathrm{~mol} / \mathrm{l}$.
Table III. Hydrolysis of poly(acrylamide $)^{\mathrm{a}}$

\begin{tabular}{cccc}
\hline$t, \min$ & $x / a$ & $t, \min$ & $x / a$ \\
\hline 5 & 0.0306 & 80 & 0.2843 \\
10 & 0.0572 & 110 & 0.3290 \\
20 & 0.1083 & 150 & 0.3725 \\
30 & 0.1491 & 200 & 0.4065 \\
45 & 0.2043 & 260 & 0.4363 \\
60 & 0.2412 & & \\
\hline
\end{tabular}

a $\mathrm{NaOH}, 0.50 \mathrm{~mol} / l$; amide, $0.025 \mathrm{~mol} / l ; 40^{\circ} \mathrm{C}$.

Table IV. Hydrolysis of poly(acrylamide $)^{a}$

\begin{tabular}{|c|c|c|c|c|c|}
\hline \multicolumn{2}{|c|}{$60^{\circ} \mathrm{C}$} & \multicolumn{2}{|c|}{$70^{\circ} \mathrm{C}$} & \multicolumn{2}{|c|}{$100^{\circ} \mathrm{C}$} \\
\hline$t, \min$ & $x / a$ & $t, \min$ & $x / a$ & $t, \min$ & $x / a$ \\
\hline 12 & 0.3117 & 12 & 0.3950 & 10 & 0.5400 \\
\hline 32 & 0.4433 & 22 & 0.4745 & 15 & 0.5690 \\
\hline 52 & 0.4965 & 32 & 0.5125 & 20 & 0.5861 \\
\hline 72 & 0.5245 & 42 & 0.5348 & 25 & 0.5955 \\
\hline 102 & 0.5503 & 52 & 0.5510 & 30 & 0.6010 \\
\hline 132 & 0.5675 & 62 & 0.5620 & 40 & 0.6072 \\
\hline 172 & 0.5835 & 82 & 0.5802 & 60 & 0.6141 \\
\hline 222 & 0.5970 & 112 & 0.5973 & 80 & 0.6181 \\
\hline 302 & 0.6100 & 152 & 0.6095 & 110 & 0.6223 \\
\hline \multirow[t]{3}{*}{407} & 0.6194 & 202 & 0.6194 & 150 & 0.6266 \\
\hline & & & & 200 & 0.6298 \\
\hline & & & & 260 & 0.6321 \\
\hline
\end{tabular}

a $\mathrm{NaOH}, 0.50 \mathrm{~mol} / l$; amide, $0.05 \mathrm{~mol} / l$.

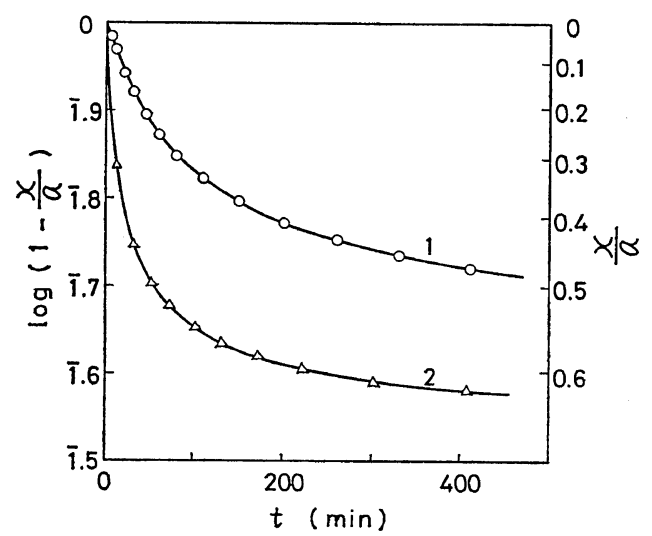

Figure 3. Pseudo first-order plots based on a simple second-order rate equation: $1, \mathrm{NaOH} 0.25$ $\mathrm{mol} / l$; amide, $0.025 \mathrm{~mol} / l, 50^{\circ} \mathrm{C} ; 2, \quad \mathrm{NaOH} 0.50$ $\mathrm{mol} / l$; amide, $0.05 \mathrm{~mol} / l, 50^{\circ} \mathrm{C}$.

several straight lines means that the reactivities of amide groups in a molecule change abruptly at the conversion degrees corresponding to the points of intersection of the lines, while they are kept constant in the regions corresponding 
Kinetic Aspects of the Alkaline Hydrolysis of Poly(acrylamide)

to the rectilinear parts. However, one can hardly develop a model to understand the above meaning. If a carboxyl group affects the neighboring groups, the amide groups in a molecule cannot be uniform in reactivity, and the plots based on the simple rate equation where the term of amide concentration is $(a-x)$ can not form any straight lines. Therefore, the results obtained by the authors may be more reasonable than the previous ones.

\section{Conversion limit}

Figure 4 shows the relations between the degree of conversion and apparent rate constants calculated by the use of eq 13 .

$$
k_{\mathrm{app}}=\frac{\mathrm{d} x / \mathrm{d} t}{(a-x)(b-x)}
$$

where $a$ and $b$ are the initial concentrations of amide and sodium hydroxide, respectively.

Judging from the tendency of the curves, we can see a critical point existing at a degree of conversion of about 0.61 . Since the ratio of the rate constant at this point to that at the start of the reaction is nealy equal to $1 / 200$ and after reaching this point the progress of the reaction slows down remarkably, we can say that a kind of conversion limit exists in the alkaline hydrolysis of poly(acrylamide) under moderate conditions, contrary to previous findings.

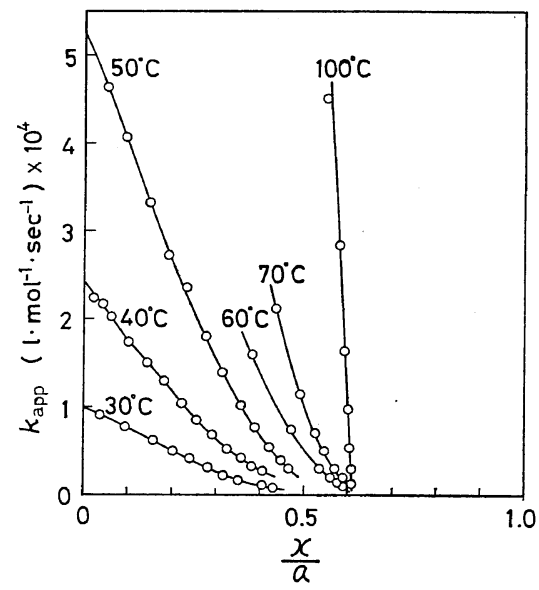

Figure 4. Relations between apparent rate constants and degree of conversion: $30 \sim 50^{\circ} \mathrm{C}, \mathrm{NaOH}$, $0.25 \mathrm{~mol} / \mathrm{l}$, amide, $0.025 \mathrm{~mol} / l ; 60 \sim 100^{\circ} \mathrm{C}$, NaOH, $0.50 \mathrm{~mol} / l$, amide, $0.05 \mathrm{~mol} / l$.
These observations confirm the validity of the assumptions adopted in the theoretical calculations, and the value, 0.61 , is considered to correspond to the critical point of conversion where the amide group II disappears.

\section{Reaction Rate in the Early Stage}

Figure 5 shows the linear relationship between the reaction time and $\log (1-2.5 x / a)$ for the reaction carried out under the ionic strength of 0.5. This result is in accord with the theory, and from this result the ratio $k_{\mathrm{II}} / k_{\mathrm{I}}$ is found to be approximately equal to the $1 / 4$ on the basis of eq $11 ; 3-2 k_{\mathrm{II}} / k_{\mathrm{I}} \fallingdotseq 2.5 \rightarrow k_{\mathrm{II}} / k_{\mathrm{I}} \fallingdotseq 0.25$.

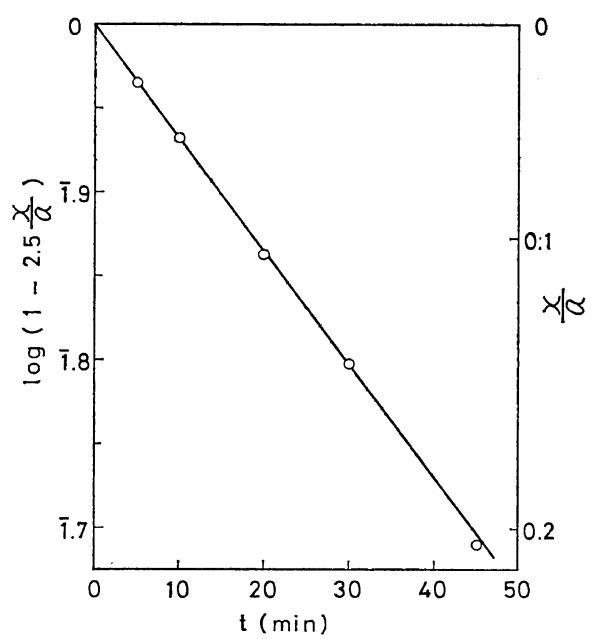

Figure 5. Pseudo first-order plot based on eq 11: $\mathrm{NaOH}, 0.50 \mathrm{~mol} / l$; amide, $0.025 \mathrm{~mol} / l ; 40^{\circ} \mathrm{C}$.

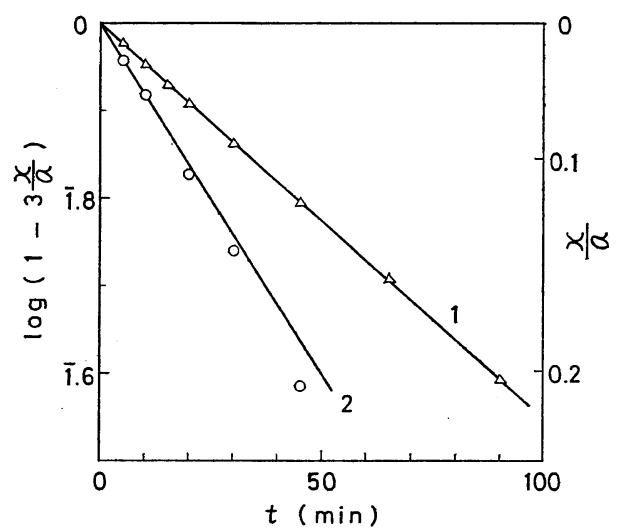

Figure 6. Pseudo first-order plots based on eq 11: $1, \mathrm{NaOH}, 0.25 \mathrm{~mol} / l, 40^{\circ} \mathrm{C} ; 2, \mathrm{NaOH}, 0.50 \mathrm{~mol} / l$, amide, $0.025 \mathrm{~mol} / l, 40^{\circ} \mathrm{C}$. 


\section{Higuchi and R. Senju}

As for the reactions under the ionic strength of 0.25 , the assumption, that only the nearest amide groups are affected by a carboxyl group, is considered to be not precise; in this case, the linear relationship was obtained between $\log (1$ $-3 x / a$ ) and the reaction time (Figure 6). If it be assumed that a carboxyl group affects not only the first neighboring groups but also the second ones, one obtains eq 14 in place of eq 11 .

$$
\frac{\mathrm{d} x}{\mathrm{~d} t}=k_{\mathrm{I}}\left[a-\left(5-\frac{2\left(k_{\mathrm{II}}{ }^{\prime}+k_{\mathrm{II}}{ }^{\prime \prime}\right)}{k_{\mathrm{I}}^{\prime}}\right) x\right]\left[\mathrm{OH}^{-}\right]
$$

where $k_{\mathrm{I}}{ }^{\prime}, k_{\mathrm{II}}{ }^{\prime}$, and $k_{\mathrm{II}}{ }^{\prime \prime}$ are the rate constants for amide groups which have no reacted groups at the first and second neighboring positions, one reacted second neighboring group, and one reacted first neighboring group, respectively. The result obtained under the ionic strength of 0.25 may

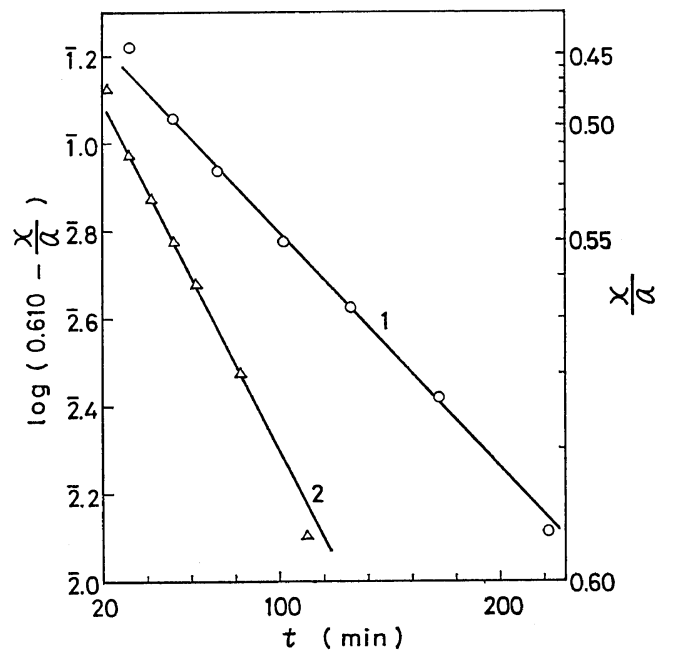

Figure 7. Pseudo first-order plots based on eq 12: 1 , $\mathrm{NaOH}, 0.50 \mathrm{~mol} / l$, amide, $0.05 \mathrm{~mol} / l, 60^{\circ} \mathrm{C} ; 2$, $\mathrm{NaOH}, 0.50 \mathrm{~mol} / l$, amide, $0.05 \mathrm{~mol} / l, 70^{\circ} \mathrm{C}$. be reasonable provided that the value for $\left(k_{\mathrm{II}}{ }^{\prime}+\right.$ $\left.k_{\mathrm{II}}{ }^{\prime \prime}\right)$ is approximately equal to the value for $k_{\mathrm{I}}{ }^{\prime}$.

Reaction Rate in the Late Stage

On the basis of eq 12, first-order plots for the reactions under the ionic strength of 0.5 are shown in Figure 7. The linear relationship between $\log (0.61-x / a)$ and the reaction time shows the validity of the assumptions adopted in the theoretical calculations.

\section{Evaluation of the Neighboring-Group Effect}

From the facts discussed above, we can conclude that the actual progress of the hydrolysis of poly(acrylamide) is in satisfactory accord with the theoretical figure, at least under the condition where sodium hydroxide is used as alkali reagent and the ionic strength is 0.5 or thereabouts.

Now the rate constants $k_{\mathrm{I}}, k_{\mathrm{II}}$, and $k_{\mathrm{III}}$ have a physical validity, and we are able to evaluate the neighboring-group effect quantitatively.

The rate constant for an amide group which is affected by neighboring carboxyl groups can be expressed by

$$
k=\frac{k T}{h} \exp \left[\frac{-\Delta G_{\text {n.N.E. }}^{\neq}}{R T}\right] \exp \left[\frac{-\Delta G_{\text {N.E. }}^{\neq}}{R T}\right]
$$

where $\Delta G_{\text {n.N.E. }}^{\neq}$is the activation free energy of the reaction in the case where amide groups are free from the neighboring-group effect, and $\Delta G_{\text {N.E. }}^{\neq}$is an increase in activation free energy due to the neighboring-group effect. Values for $\Delta G_{\mathrm{N} . \mathrm{E}}^{*}$ are calculated from the ratios, $k_{\mathrm{II}} / k_{\mathrm{I}}$ and $k_{\mathrm{III}} / k_{\mathrm{I}}$, on the basis of eq 15. As an example, values for $k_{\mathrm{I}}, k_{\mathrm{II}}, k_{\mathrm{III}}$, and $\Delta G_{\mathrm{N} . \mathrm{E}}^{\neq}$. under the ionic strength of 0.5 at $60^{\circ} \mathrm{C}$ are shown in Table $\mathrm{V}$.

The effect of a carboxyl group on the reactivities of neighboring amide groups is considered

\begin{tabular}{|c|c|c|c|c|}
\hline $\begin{array}{l}\text { Situation } \\
\text { A, amide } \\
\text { C, carboxyl }\end{array}$ & $\begin{array}{c}-\mathrm{A}-\mathrm{A}-\mathrm{A}- \\
\vdots\end{array}$ & $\begin{array}{cc}-\mathrm{A}-\mathrm{A}-\mathrm{C}-\mathrm{A}-\mathrm{A}- \\
\vdots & \vdots\end{array}$ & $\begin{array}{c}-\mathrm{C}-\mathrm{A}-\mathrm{A}-\mathrm{C}- \\
\vdots \vdots\end{array}$ & $\begin{array}{c}-\mathrm{C}-\mathrm{C}-\mathrm{A}-\mathrm{C}- \\
\vdots\end{array}$ \\
\hline $\begin{array}{l}\text { Rate constant, } \\
\times 10^{-4} l \mathrm{~mol}^{-1} \mathrm{sec}^{-1}\end{array}$ & $\begin{array}{c}k_{\mathrm{I}} \\
(10.8)^{\mathrm{b}}\end{array}$ & $\begin{array}{c}k_{\mathrm{II}} \\
3\end{array}$ & $\begin{array}{l}k_{\mathrm{II}} \\
2\end{array}$ & $\begin{array}{c}k_{\mathrm{III}} \\
0.05\end{array}$ \\
\hline$\Delta G_{\mathrm{N} . \mathrm{E} .}^{\neq}, \mathrm{cal} / \mathrm{mol}$ & 0 & 700 & 1000 & 3000 \\
\hline
\end{tabular}

Table V. Effect of carboxyl groups on the reactivities of neighboring amide groups ${ }^{a}$

a $\mathrm{NaOH}, 0.5 \mathrm{~mol} / \mathrm{l} ; 60^{\circ} \mathrm{C}$.

b Calculated from the data on the reactions in $0.25-N \mathrm{NaOH} ; k_{\mathrm{I}}=0.99\left(30^{\circ} \mathrm{C}\right), 2.30\left(40^{\circ} \mathrm{C}\right)$, and 5.10 $\left(50^{\circ} \mathrm{C}\right)\left(\times 10^{-4} l \mathrm{~mol}^{-1} \mathrm{sec}^{-1}\right)$; activation energy $(E)=16.0 \mathrm{kcal} / \mathrm{mol}$. 
Kinetic Aspects of the Alkaline Hydrolysis of Poly(acrylamide)

to be due to the electrostatic repulsion between the ionized group and catalyzing hydroxyl ions. When a hydroxyl ion forms the transition state complex with an amide group which is fixed at a point of distance, $r$, from a carboxyl ion, additional work $e \psi_{(r)}$ has to be done, where $\psi_{(r)}$ is time-averaged electrostatic potential at the point, and this work $\left(e \psi_{(r)}\right)$ may correspond to the increase in the activation free energy $\left(\Delta G_{\mathrm{N} . E .}^{\neq}\right)$, i.e., $e \psi_{(r)}=(k / R) \Delta G_{\mathrm{N} . E .}^{ \pm}$.

The distance $\left(\gamma_{\alpha, \beta}\right)$ between a carboxyl group $(\alpha)$ and a nearest-neighboring amide group $(\beta)$ differs as the case may be: in the case where $\alpha$ and $\beta$ are arranged in phase, $\gamma_{\alpha, \beta}$ varies within the range of $2.5 \sim 3.5 \AA$; while in the case where $\alpha$ and $\beta$ are arranged zigzag $\gamma_{\alpha, \beta}$ varies within the range of $3.8 \sim 4.2 \AA$. If it be assumed that the average value for $\gamma_{a, \beta}$ is equal to $3.5 \AA$, we obtain a result, from eq 16 and 17 , that the time-averaged electrostatic potential at a point $3.5 \AA$ distant from a carboxyl group in $0.5-N$ sodium hydroxide solution at $60^{\circ} \mathrm{C}$ is nearly equal to one half of that in an extremely diluted solution.

$$
\begin{aligned}
\psi_{r=3.5 \AA}, I \doteqdot 0,60^{\circ} \mathrm{C} & =\frac{e}{\varepsilon r}=\frac{4.8 \times 10^{-10}}{66.5 \times 3.5 \times 10^{-8}} \\
& =2.06 \times 10^{-4}(\mathrm{esu}) \\
\psi_{r=3.5 \AA}, I=0.5,60^{\circ} \mathrm{C} & =\frac{k \Delta G_{\mathrm{N} . \mathrm{E} .}^{\neq}}{e R}=\frac{1.38 \times 10^{-10} \times 700}{4.8 \times 10^{-10} \times 1.986} \\
& =1.01 \times 10^{-4}(\mathrm{esu})
\end{aligned}
$$

This result appears to be valid on the basis of the above experiment.

\section{REFERENCES}

1. C. L. Arcus, J. Chem. Soc., 2732 (1949).

2. J. Moens and G. Smets, J. Polym. Sci., 23, 931 (1957).

3. S. H. Pinner, ibid., 10, 376 (1953).

4. J. B. Keller, J. Chem. Phys., 37, 2584 (1962), ibid., 38, 325 (1963); T. Alfrey, Jr., and W. G. Lloyd, ibid., 38, 318 (1963); C. B. Arends, ibid., 38, 322 (1963); L. Lazare, ibid., 39, 727 (1963). 